\title{
Avaliação da concentração de fluoreto em água de abastecimento de Centros Municipais de Educação Infantil: um estudo exploratório
}

Adriano Pereira Ramiro'; Christiane de Fátima Sá Sousa²; Rejane de Oliveira Teixeira ${ }^{3}$; Ovidia Augusta da Fonseca Almeida Brito4; Leandro Brambilla Martorel5; Lidia Moraes Ribeiro Jordão6.

${ }^{1}$ Mestrando em Ciências Moleculares UEG. Químico responsável técnico no Centro Universitário de Anápolis - UniEVANGÉLICA; ${ }^{2}$ Especialista em Engenharia de Produção Centro Universitário de Anápolis - UniEVANGÉLICA; ${ }^{3}$ Especialista em Tecnologia em Química Industrial - Faculdade de tecnologia SENAI Roberto Mange; ${ }^{4}$ Especialista em Gestão Estratégica de Marketing, Professora Faculdade de tecnologia SENAI Roberto Mange; ${ }^{5}$ Doutor em Bioética pela UnB, Professor do Curso de Odontologia do Centro Universitário de Anápolis - UniEVANGÉLICA e da Faculdade de Odontologia da Universidade Federal de Goiás; ${ }^{6}$ Doutora em Odontologia pela UFG, Professora da Faculdade de Odontologia da Universidade Federal de Goiás.

\begin{abstract}
Resumo
Introdução: A concentração de fluoreto é um parâmetro importante para a avaliação da qualidade de fluoretação das águas de abastecimento, sendo o teor recomendado de 0,6 a 0,8 ppm. Objetivo: avaliar a concentração de fluoreto nas águas que abastecem dez Centros Municipais de Educação Infantil (CMEIs) no município de Anápolis. Métodos: avaliações realizadas por meio da análise do íon flúor nas amostras estudadas, utilizando colorímetro digital para as leituras. Resultados: Oito dos dez CMEIs apresentaram amostras dentro das especificações esperadas. Os dois CMEIs que apresentaram valores abaixo do recomendado utilizavam água proveniente de poço, cisterna ou fontes mistas. Conclusões: Os dados obtidos neste estudo reforçam a importância do monitoramento da concentração de fluoreto na água consumida em instituições de ensino, uma vez que a população infantil constitui grupo de risco para o desenvolvimento de fluorose dentária.
\end{abstract}

Palavras-chave: Abastecimento de água; Fluoretação; Monitoramento.

\section{Introdução}

Nas últimas décadas, o flúor foi uma das medidas de maior impacto referente ao controle da cárie dentária, já que este, em concentrações adequadas disponíveis no meio bucal ${ }^{1}$, interfere diretamente na reação de desmineralização e remineralização dentária. Por ser um método de grande abrangência, beneficiar a todos os grupos socioeconômicos e ter excelente relação custo-benefício ${ }^{1-3}$, a fluoretação da água foi reconhecida como um dos dez avanços mais importantes para a saúde pública do século XX e recomendada pela Organização Mundial da Saúde (OMS).

Assim, a concentração de fluoreto é um parâmetro importante para a avaliação da qualidade das águas de abastecimento. No Brasil, a fluoretação da água passou a ser obrigatória a partir da promulgação da Lei no. 6.050/1974. As normas e padrões nacionais para a fluoretação da água dos sistemas públicos de abastecimento destinado ao consumo humano foram estabelecidos pela Portaria $n^{\circ}$. 635/ Bsb/1975 do Ministério da Saúde, tendo como referência as médias das temperaturas máximas anuais, em virtude da previsão da quantidade de água ingerida durante o dia. Dessa forma, definiu-se a concentração de íon fluoreto, situada entre 0,6 e 0,8 ppm, como a recomendada para maximizar a prevenção de cárie dentária e limitar a ocorrência de fluorose dentária ${ }^{3-8}$.

O flúor, além de presente na água e nas pastas de dente, pode ainda ser adicionado a alimentos e bebidas industrializados, o que contribui para uma ingestão concomitante de fluoretos por diferentes fontes. É importante ressaltar que o período crítico de exposição a doses excessivas de fluoreto é do nascimento até os oito anos de idade e seu consumo de forma prolongada com concentração excessiva pode resultar em fluorose, condição que promove mudanças nos ossos e/ou dentes, além de ossificação de tendões e ligamentos ${ }^{6,9-11}$. A evidenciação clínica da fluorose dentária é dada pela presença de manchas ou linhas opacas, bilaterais, homólogas, simétricas desde as formas mais brandas, cuja coloração é esbranquiçada até as mais graves, acastanhadas, ou com perda de estrutura ${ }^{12}$.

Dada a relevância da fluoretação das águas para a saúde pública, mais especificamente para a prevenção da cárie e potencial risco de fluorose dentária, é importante que haja um sistema capaz de monitorar e avaliar a concentração de flúor em todo o território nacional. De modo mais geral, foi implantado pelo Ministério da Saúde, no ano 2000, o Programa de Vigilância em Saúde Ambiental relacionada à Qualidade da Água para Consumo Humano - Vigiagua -, integrante do Subsistema Nacional de Vigilância Ambiental em Saúde, que tornou mais evidente a responsabilidade do setor saúde em operacionalizar estratégias para este controle. A partir de então, a fluoretação das águas encontrou um espaço com legitimidade para garantir o cumprimento dos padrões estabelecidos na legislação vigente $e^{7,13,14}$.

Dentro do setor da odontologia, em 2006, - Ministério da Saúde instituiu o Comitê Técnico Assessor (CTA) que passou a contribuir com a Coordenação Nacional de Saúde Bucal no que diz respeito às estratégias e diretrizes para a Vigilância em Saúde Bucal. Uma importante iniciativa do CTA foi a criação do Centro Colaborador do Ministério da Saúde em Vigilância da Saúde Bucal (CECOL), em algumas universidades brasileiras, no intuito de apoiar as ações de vigilância em saúde bucal ${ }^{15}$. 
Dentre as ações desenvolvidas pelo CECOL, destacam-se as relacionadas ao heterocontrole da fluoretação das águas de abastecimento público. Entre os anos de 2010 e 2015, o CECOL/USP coordenou uma pesquisa intitulada "Cobertura e vigilância da fluoretação da água de abastecimento público no Brasil", mais conhecida como "Projeto Vigifluor", e que trabalhou em rede com os demais CECOL do Brasil. Tal pesquisa buscou "mapear a cobertura da fluoretação das águas de abastecimento público nos 614 municípios brasileiros com mais de 50 mil habitantes (...) e identificar (...) aqueles em que se realizam ações de vigilância da fluoretação"16.

Considerando-se a importância do monitoramento periódico da presença no fluoreto na água de abastecimento bem como a escassez de estudos dessa natureza na região Centro-Oeste, o objetivo do presente trabalho foi realizar uma avaliação exploratória da concentração de fluoreto na água que abastece dez Centros Municipais de Educação Infantil (CMEIs), popularmente conhecidos como "creches", no município de Anápolis.

\section{Material e Métodos}

Este estudo observacional exploratório foi realizado no município de Anápolis, estado de Goiás, durante os meses de novembro e dezembro de 2015. A escolha dos elementos da amostra foi feita de forma não-aleatória, intencional e por conveniência ${ }^{17}$. Antes da coleta de dados, foi realizado contato formal com os responsáveis pelas respectivas instituições de ensino infantil, solicitando permissão de acesso aos locais e informando os objetivos da pesquisa em relação à identificação da concentração de fluoretos na água e sua repercussão para a saúde bucal das crianças.

A cidade de Anápolis possui duas Estações de Tratamento de Água (ETA). Nesta pesquisa todos os CMEIs avaliados recebiam água tratada de modo rotineiro de uma destas.

A coleta das amostras foi realizada nos bebedouros das instituições, baseando-se no Protocolo de Coleta e Análise da Amostra de Água do Projeto Vigifluor ${ }^{2}$, utilizando-se frascos de polietileno de $100 \mathrm{~mL}$. Esses frascos foram identificados com informações de hora, nome, endereço do local e fonte da água coletada, e posteriormente foram acondicionados e encaminhados para análise.

A análise da concentração de flúor foi realizada no laboratório de química do Centro Universitário de Anápolis - UniEVANGÉLICA, utilizando um Colorímetro digital da marca Quimis ${ }^{\circledR}$ modelo Q406CFF, previamente calibrado seguindo a metodologia do manual de instruções fornecido pelo fabricante. A amostragem de alíquota de $10 \mathrm{~mL}$ foi acondicionada com auxílio de uma seringa dentro da cubeta, onde foram adicionados $2 \mathrm{~mL}$ da solução de SPANDS, fornecida no Kit de análise do fabricante, realizando a homogeneização suave desta solução. Esta reação baseia-se na reação entre o fluoreto e o corante zircônio, o fluoreto atua com o corante, dissociando uma porção deste em um complexo aniônico sem cor (ZrF62-). A quantidade de fluoreto é inversamente proporcional à cor produzida, ou seja, se torna progressivamente mais clara enquanto a concentração de fluoreto aumenta.

Após o procedimento citado e a limpeza da cubeta, foi realizada a leitura observando o resultado fornecido em ppm (parte por milhão). Os valores obtidos nas leituras das amostras em triplicata foram repassados para uma planilha eletrônica no programa Microsoft Excel ${ }^{\circledR}$ e analisados por estatística descritiva.

\section{Resultados}

Nas dez amostras avaliadas, os valores referentes à concentração de fluoreto variaram de 0,00 mg F/L a 0,81mg F/L, conforme apresentado na Tabela 1 . Tem-se que a melhor relação risco-benefício para municípios com médias de temperatura similares às de Anápolis está entre o teor de 0,55 a 0,84 $\mathrm{mg} F / \mathrm{L}$, em que o benefício é máximo e o risco é baixo (Quadro 1). Assim, oito das dez amostras estavam em conformidade com os critérios estabelecidos.

Tabela 1 - Média e desvio-padrão (DP) das concentrações de flúor em CMEIs. Anápolis - GO, 2015.

\begin{tabular}{cccc}
\hline $\begin{array}{c}\text { Amostras/ } \\
\text { CMEI }\end{array}$ & $\begin{array}{c}\text { Fonte de } \\
\text { abastecimento do CMEI }\end{array}$ & Média (mg F/L) & $\begin{array}{c}\text { Desvio- } \\
\text { padrão }\end{array}$ \\
\hline A & Pública & 0,77 & 0,0058 \\
B & Pública & 0,81 & 0,0058 \\
C & Pública & 0,77 & 0,0115 \\
D & Pública & 0,70 & 0,0115 \\
E & Pública & 0,80 & 0,0115 \\
F & Pública & 0,75 & 0,0058 \\
G & Pública & 0,64 & 0,0115 \\
H & Mista & 0,26 & 0,0173 \\
I & Local & 0,00 & 0,0000 \\
J & Pública & 0,80 & 0,0231 \\
\hline
\end{tabular}

Mais especificamente, as amostras B, E e J estavam próximas do limítrofe superior do padrão estabelecido e a amostra B apresentava maior concentração de flúor (0,81mg F/L). Já as amostras H e I apresentaram valores abaixo da especificação. No caso da amostra I, a água utilizada era somente de poço artesiano, o que explica a ausência de flúor na amostra. $\mathrm{Na}$ amostra $\mathrm{H}$, o valor bem abaixo da especificação se explica pela mistura da água da rede de abastecimento público e da água do poço artesiano (mista), diluindo o íon flúor na amostra.

\section{Discussão}

Observou-se que $80 \%$ dos teores de flúor nas amostras analisadas ofereciam o benefício máximo no combate à cárie dentária. Desse modo, as concentrações de flúor na água dos CMEIs (creches) que informaram como fonte única a rede de abastecimento pública, estavam dentro dos valores considerados ideais, segundo os padrões estabelecidos.

Sabe-se que a temperatura ambiental deve ser considerada quando se avalia o consumo de água fluoretada, uma vez que em clima tro- 
pical as pessoas tendem a consumir mais água, por transpirarem mais. Além disso, atualmente ocorrem constantes mudanças climáticas, elevadas temperaturas e episódios frequentes de escassez de água, realidade bem diferente dos registros de 50 anos atrás ${ }^{18}$. Assim, no período entre 2009 e 2011, diversos pesquisadores brasileiros experts em vigilância da fluoretação das águas mantiveram discussões sobre da em estudo realizado em Campo Alegre de Lourdes-BA8, onde as águas de poços da região não possuíam níveis de fluoreto suficientes para prevenção da cárie dentária. Segundo estimativas do Vigifluor, a população coberta com água tratada e fluoretada no município de Anápolis corresponde a 92,5\% (334.977 indivíduos) ${ }^{16}$. O Vigifluor divulgou ainda que em 2015, 83\% das amostras no estado de São Paulo estavam na fai-

Quadro 1 - Teor de flúor para localidades com médias de temperaturas máximas entre 26,3 e $32,5^{\circ} \mathrm{C}$.

\begin{tabular}{|c|c|c|}
\hline $\begin{array}{l}\text { Teor de flúor na água } \\
\text { (em ppm ou } m g F / L)\end{array}$ & $\begin{array}{c}\text { Benefício } \\
\text { (prevenir cárie) }\end{array}$ & $\begin{array}{c}\text { Risco } \\
(\text { produzir fluorose dentária) }\end{array}$ \\
\hline 0,00 a 0,44 & Insignificante & Insignificante \\
\hline 0,45 a 0,54 & Mínimo & Baixo \\
\hline 0,55 a 0,84 & Máximo & Baixo \\
\hline 0,85 a 1,14 & Máximo & Moderado \\
\hline 1,15 a 1,44 & Questionável & Alto \\
\hline 1,45 ou mais & Malefício & Muito alto \\
\hline
\end{tabular}

Fonte: CECOL, 2011.

o teor ideal de flúor na água. A classificação do teor de flúor em apenas duas categorias (adequado e não adequado) foi então considerada inapropriada, por reduzir as possibilidades de interpretação e atribuição de significado às amostras coletadas em diferentes municípios brasileiros. Considerando que a fluoretação das águas pode trazer benefício (prevenir cárie) e risco (produzir fluorose dentária), estabeleceu-se uma classificação do teor de flúor da água de acordo com estes dois critérios, incorporando a informação sobre a média da temperatura máxima anual do local avaliado ${ }^{19}$.

O Instituto Nacional de Meteorologia não disponibiliza dados de todos os municípios brasileiros, entretanto, considerando a média da temperatura máxima anual de municípios próximos à Anápolis para o ano de 2015, como Goiânia e Pirenópolis, identificou-se que Anápolis se enquadraria nos grupos de municípios com médias entre $26,3^{\circ} \mathrm{C}$ e 32,5 ${ }^{\circ} \mathrm{C}$. Dessa forma, o consenso técnico com a classificação de águas de abastecimento público segundo o teor de flúor para esta variação de média de temperatura máxima é apresentado no quadro 1, o que permite a interpretação dos achados deste estudo. É importante lembrar que a presente avaliação foi realizada em um período crítico de falta de água, onde várias regiões do país enfrentaram problemas com abastecimento, o que leva a maior parte de seus habitantes a consumir água sem tratamento, provenientes de poços e cisternas. Situação semelhante foi encontra- xa ideal em termos de prevenção à cárie ${ }^{20}$. No site do CECOL/USP é possível acessar o laudo de análise do íon flúor do ano de 2014 para o município de Anápolis que integrou a referida pesquisa. Das 24 amostras analisadas, 16 (75\%) estavam na faixa ideal para prevenção de cárie e baixo risco para fluorose dentária, resultados próximos do encontrado nos CMEIs avaliados. Ressalta-se que o presente estudo precisaria ser extrapolado para outros pontos de coleta e por um maior período de tempo para se afirmar que o abastecimento público do município de Anápolis atende de fato aos valores ideais de fluoretação. O número reduzido de amostras e a pontualidade da investigação constituem suas principais limitações. Por outro lado, visto que a fluoretação das águas é uma questão de saúde pública, o monitoramento da qualidade da água de abastecimento público por instituições não envolvidas nos procedimentos de fluoretação garante a imparcialidade e credibilidade dos resultados obtidos. No momento, ainda são poucos os estudos disponíveis sobre a fluoretação de águas na região Centro-Oeste, ao contrário de outras regiões do país 1,3, 21-23.

\section{Conclusão}

As oito instituições que utilizavam água do abastecimento público apresentaram concentrações de fluoreto dentro dos padrões recomendados para prevenção da cárie dentária e abaixo da concentração 
de risco para fluorose dentária. Já as duas instituições de ensino que utilizavam água proveniente de poço, cisterna, ou fontes mistas apresentaram concentrações de fluoreto abaixo do valor suficiente para a prevenção da cárie. O presente estudo reforça a importância do monitoramento das concentrações de íons fluoreto na água consumida em instituições de ensino, uma vez que a população infantil constitui grupo de risco para a fluorose dentária. Recomenda-se que medidas de monitoramento da fluoretação a nível local sejam implementadas, com vistas a fortalecer o sistema de vigilância municipal, e que a rede de abastecimento público de água seja expandida para incluir as instituições descobertas.

\section{Referências}

1. Moimaz SAS, Saliba O, Garbin CAS, Garbin AJI, Sumida DH, Chiba FY, et al. Fluoretação das águas de abastecimento público no município de Araçatuba. Rev. Odontol. Araçatuba (Online) 2012; 33(1):54-60

2. Centro Colaborador do Ministério da Saúde em Vigilância da Saúde Bucal (CECOL), da Universidade de São Paulo (USP). Protocolo de coleta e análise da amostra de água Projeto Vigifluor: cobertura e vigilância da fluoretação das águas de abastecimento público no Brasil. São Paulo, 2014.

3. Frazão $P$, Soares CCS, Fernandes GF, Marques RAA, Narvai PC. Fluoretação da água e insuficiências no sistema de informação da política de vigilância à saúde. Rev. Assoc. Paul. Cir. Dent. 2013; 67 (2):94-100.

4. Ministério da Saúde (Brasil). Portaria $n^{\circ}$. 635, de 26 de dezembro de 1975. Aprova normas e padrões, a seguir, sobre a fluoretação da água dos sistemas públicos de abastecimento, destinada ao consumo humano. Diário Oficial da União 30 jan 1976.

5. Silva RA, Petrarca MH, Santos RC, Yamamoto IT, Marques LRM. Monitoramento da concentração de íons fluoreto na água destinada ao consumo humano proveniente de um município com diferentes sistemas de abastecimento público. Ver. Inst. Adolfo Lutz (Impr.). 2011; 70(2):220-224.

6. Buendia OC. Fluoretação de Águas: Manual de Orientação Prática. São Paulo: Editora American Med; 1996.

7. Cesa K, Abegg C, Aerts D. A vigilância da fluoretação de águas nas capitais brasileiras. Epidemiol. Serv. Saúde. 2011; 20(4):547-555

8. Moura MS, Gomes LMA, Castro MRP, Teles JBM, Moura LFAD. Fluorose dentária em escolares de 12 anos. Rev. Gaúcha Odontol. 2010; 58(4):463-468.

9. Costa AB, Lobo EA, Kirst A. Estudo comparativo da concentração de flúor, pH e condutividade elétrica da água subterrânea dos municípios de Santa Cruz do Sul, Venâncio Aires e Vera Cruz, Brasil. In anais do XIII Congresso Brasileiro de Águas Subterrâneas, São Paulo, pp 2-3, Out. 2004

10. CataniI DB, Hugol FN, Cypriano IS, Sousa MLR, Cury JA. Relação entre níveis de fluoreto na água de abastecimento públi- co e fluorose dental. Rev. Saúde Públ. 2007;41(5):732-39.

11. Jordão LMR, Vasconcelos DN, Moreira RS, Freire MCM. Fluorose dentária: prevalência e fatores associados em escolares de 12 anos de Goiânia, Goiás. Rev. Bras. Epidemiol. 2015;18(3):568577.

12. Spohr AR, Menegaz AM, Favetti M, Flores RZ, Horn T, Benetti $T$ et al. Prevalência de fluorose dentária em adolescentes de escolas municipais da área urbana do município de Pelotas/RS. Rev. Fac. Odontol. 2010; 51(3):5-10.

13. Ministério da Saúde. Secretaria de Vigilância em Saúde. Coordenação-Geral de Vigilância em Saúde Ambiental (Brasil). Programa Nacional de Vigilância em Saúde Ambiental relacionada à qualidade da água para consumo humano. Brasília, 2005.

14. Ministério da Saúde. Secretaria de Vigilância em Saúde. Coordenação-Geral de Vigilância em Saúde Ambiental (Brasil). Diretriz Nacional do Plano de Amostragem da Vigilância Ambiental em Saúde Relacionada à Qualidade da Água para consumo humano. Vigiagua. Brasília, 2005.

15. Moysés SJ, Pucca Junior GA, Paludetto Junior M, Moura L. Avanços e desafios à Política de Vigilância à Saúde Bucal no Brasil. Rev. Saúde Públ. 2013; 47(Suppl 3):161-167.

16. Frazão $P$, Narvai PC. Cobertura e vigilância da fluoretação da água no Brasil: municípios com mais de 50 mil habitantes. São Paulo: Faculdade de Saúde Pública da USP, 2017. 202 p.

17. Fink A. How to sample in surveys. Sage: Thousand Oaks; 1995.

18. Frazão P, Peres MA, Cury JA. Padrões de potabilidade da água para consumo humano quanto ao teor de flúor: subsídios para a revisão da Portaria MS 518/2004. Parecer Técnico-Científico, São Paulo, 2010.

19. Centro Colaborador do Ministério da Saúde em Vigilância da Saúde Bucal (CECOL). Consenso técnico sobre classificação de águas de abastecimento público segundo o teor de flúor. São Paulo: Faculdade de Saúde Pública da Universidade de São Paulo, 2011.

20. Revista do Conselho Regional de Odontologia de São Paulo (CROSP). 2016. Ano III; 04:34

21. Frazão $P$, Peres MA, Cury JA. Qualidade da água para consumo humano e concentração de fluoreto. Rev. Saúde Públ. 2011;45(5):964-73.

22. Peixoto DF, Alencar KP, Peixoto RF, Sousa CFM, Sampaio DC Forte FDS. Heterocontrole da fluoretação da água de abastecimento público do município de Jaguaribara, Ceará, Brasil. Rev. Bras. Promoç. Saúde. 2012;25(3): 271-277.

23. Bernardes CF. Fluoretação da água destinada ao consumo humano no município de Campinas. Biokos. 2005:19(1/2):39-43.

Recebido em : 24/07/2018

Aprovado em: 10/10/2018

Os autores declaram que não há conflitos de interesse. 


\title{
Autor Correspondente:
}

Adriano Pereira Ramiro*

Químico responsável técnico no Centro Universitário de

Anápolis - UniEVANGÉLICA; Laboratório de Química.

Bloco C. $2^{\circ}$ andar. Avenida Universitária Km. 3, 5 - Cida-

de Universitária, Anápolis - GO, 75083-515.

Telefone: +55 (62) 9135-1139

E-mail: nano.quimica@hotmail.com

\section{Evaluation of fluoride concentration in water supply of nursery schools: an ex- ploratory study}

\begin{abstract}
Introduction: Fluoride concentration is an important parameter for the quality assessment of water fluoridation, being the recommended content of fluoride between 0.6 and $0.8 \mathrm{ppm}$. Aim: The aim of this study was to evaluate the fluoridation of the water supplying 10 creches in Anápolis, through fluoride analysis in a digital colorimeter. Methods: Analysis of the flow of samples using digital colorimeter. Results: Eight of the ten samples, each representing one creche, were within the expected specifications. The remaining two creches used water from either mixed sources or wells. Conclusion: The data obtained in this study highlights the importance of monitoring the fluoride concentration in water serving nursery schools, since children constitute a risk group for the development of dental fluorosis.
\end{abstract}

Keywords: Water supply; Fluoridation; Monitoring. 